\title{
Bone Turnover and Growth during and after Chemotherapy in Children with Solid Tumors
}

\author{
LOUISE E. BATH, PATRICIA M. CROFTON, AGNES E.M. EVANS, MICHAEL B. RANKE, \\ MARTIN W. ELMLINGER, CHRISTOPHER J.H. KELNAR, AND W. HAMISH B. WALLACE \\ Section of Child Life and Health [L.E.B., P.M.C., A.E M.E., C.J.H.K., W.H.B.W.], Department of \\ Reproductive and Developmental Sciences, Edinburgh EH9 IUW, Scotland; Department of Paediatric \\ Biochemistry [P.M.C.], Royal Hospital for Sick Children, Sciennes Road, Edinburgh EH9 ILF, Scotland; \\ and University Children's Hospital [M.B.R., M.W.E.], Tubingen, Germany
}

\begin{abstract}
Children who are treated for malignancy have been shown to have decreased bone mineral density. We investigated the effect of serial courses of chemotherapy on growth and bone turnover in children with solid tumors. We measured height, weight, and lower leg length (LLL; $n=10$ ) and markers of bone formation [bone alkaline phosphatase (BALP) and C-terminal propeptide of type I collagen $(\mathrm{P} 1 \mathrm{CP})]$, bone resorption [C terminal telopeptide of type I collagen (1CTP)], soft tissue collagen turnover [Nterminal propeptide of type III procollagen (P3NP)], and the $\mathrm{GH}$ axis [IGF1 and its binding proteins (IGFBP3 and IGFBP2)] before and after each course $(n=25)$ and on completion of treatment $(n=12)$. Height SD score decreased during treatment $(p<0.01)$ and increased to pretreatment levels at 3 mo off treatment $(p<0.05)$. LLL growth increased off treatment $(p<$ 0.01). At diagnosis, BALP, PICP, and IGF1 SD score were low compared with age- and sex-matched reference groups $(p<$ $0.001, p<0.001$, and $p<0.002$, respectively) and IGFBP2 was elevated ( $p<0.001$ ). During treatment, P1CP, 1CTP, and P3NP showed a cyclical pattern decreasing after each course $(p<$
\end{abstract}

Impaired growth is a complication of the treatment of neoplastic disease in children (1-3). Factors that may contribute to poor growth during treatment include the underlying disease process, chemotherapy, irradiation, concurrent serious infection, and poor nutrition. Increasing evidence suggests that cytotoxic chemotherapy has a significant detrimental effect on bone turnover and growth during treatment (4-9). The risk of developing osteoporosis is unclear, and the long-term effects on bone mineralization and bone strength are not known.

Children with acute lymphoblastic leukemia have low bone turnover and are in a GH-resistant state at diagnosis. Steroid therapy has been shown to suppress osteoblast proliferation,

Received July 26, 2002; accepted May 5, 2003.

Correspondence: Louise E. Bath, PhD, Section of Child Life and Health, Department of Reproductive and Developmental Sciences, 20 Sylvan Place, Edinburgh EH9 IUW, Scotland; e-mail: louise.bath@luht.scot.nhs.uk

Supported by Serono Laboratories, the Child Growth Foundation and the Jennifer Fund.

DOI: 10.1203/01.PDR.0000100903.83472.09
$0.001)$ and increasing before the next course $(p<0.001)$. Precourse levels of BALP, P1CP, 1CTP, P3NP, IGF1, and IGFBP3 showed an upward trend during treatment. BALP remained suppressed throughout treatment $(p<0.001)$. Intense courses of treatment for solid tumors have a direct suppressive effect on bone turnover, with an imbalance between collagen synthesis and degradation. (Pediatr Res 55: 224-230, 2004)
Abbreviations
BALP, bone alkaline phosphatase
BMI, body mass index
1CTP, C-terminal telopeptide of type I collagen
IGFBP, IGF-binding protein
LLL, lower leg length
LLLV, lower leg length velocity
P1CP, C-terminal propeptide of type I collagen
P3NP, N-terminal propeptide of type III procollagen
SDS, standard deviation score

osteoclast activity, and lower leg growth (5). High-dose methotrexate as CNS treatment has been shown to reduce bone formation and enhance bone resorption (4). Children with solid tumors have also been demonstrated to grow slowly during treatment with cytotoxic agents (10), but there have been few studies on bone turnover in this group of patients.

Growth is a dynamic process with daily, weekly, and monthly fluctuations in velocity. Height measurements give a reliable indicator of growth velocity over periods longer than 6 mo. Short-term changes in growth may be measured by knemometry in children who are compliant (5). Changes in lower leg length (LLL) may be assessed frequently and give an indication of whether interventions are affecting growth. Biochemical markers of growth and bone turnover reflect the GH axis, bone modeling, and, indirectly, activity at the growth plate.

The aim of this study was to investigate the effect of intensive courses of chemotherapy on growth and bone turn- 
over in children who have a diagnosis of solid tumors requiring treatment with intensive cytotoxic agents. Our hypothesis was that any such effects are a direct effect of the chemotherapeutic agents used, rather than part of the disease process, poor nutrition, or an indirect effect on the GH axis. We assessed anthropometric indices at diagnosis and during and on completion of treatment and measured markers of the GH axis and markers of bone and soft tissue formation and resorption before and after each course of treatment and on completion of therapy. We determined whether there was evidence of a decrease in growth rate during treatment, catch-up in growth on completion of therapy, biochemical evidence of changes in bone turnover, and whether these changes could be attributed to the cytotoxic therapy.

\section{METHODS}

\section{Subjects}

We studied 25 children who had a diagnosis of a solid tumor requiring cytotoxic therapy. All patients who presented to the Royal Hospital for Sick Children between June 1996 and January 1998 with a solid tumor requiring intensive courses of chemotherapy were eligible to participate in the study. During the $18 \mathrm{mo}, 25$ patients presented and all agreed to participate. The clinical details of patients at diagnosis, drug therapy, and outcome are detailed in Table 1. All children were treated according to nationally agreed protocols. The intensive chemotherapy was administered three times weekly, except for those children who were randomized to received rapid carboplatin, vincristine, cisplatin, etoposide, cyclophosphamide
(COJEC) (every $10 \mathrm{~d}$ ) or the intensive arm of the osteosarcoma protocol (every $14 \mathrm{~d}$ ) and the one patient who initially was treated on the infant brain protocol, who received treatment fortnightly. All children were treated with intensive chemotherapy requiring antiemetic therapy and had significant bone marrow suppression. During chemotherapy, the patients received antiemetic therapy, either ondansetron $\left(5 \mathrm{mg} / \mathrm{m}^{2} / \mathrm{d}\right)$ alone or ondansetron and dexamethasone $\left(4 \mathrm{mg} / \mathrm{m}^{2} / \mathrm{d}\right.$; see Table 1). Patients who received only ondansetron had less emetogenic protocols and included those who were treated for non-Hodgkin's lymphoma, who received high doses of prednisolone for courses $1-3$ and course 6 of their treatment.

Two patients withdrew from the study: one patient with a Wilms' tumor, at the parents' request, as the sampling from her central access venous device caused too much distress, and one patient as chemotherapy was discontinued and she received craniospinal irradiation.

\section{Consent}

Informed written consent to perform the study was obtained from the parents and from the patients when they were able to give informed consent. The local ethics committee gave their approval for the study.

\section{Samples and Anthropometric Measurements}

We measured height, weight, and LLL (by knemometry) on all prepubertal children older than $5 \mathrm{y}(n=10)$ at diagnosis and at the start of each course of treatment $(n=10)$ and monthly for 3 mo on completion of therapy $(n=9)$. Knemometry

Table 1. Clinical details of patients: at diagnosis, the treatment schedule, antiemetic medication and outcome

\begin{tabular}{|c|c|c|c|c|c|c|}
\hline Diagnosis & Stage at diagnosis & Age (y) at diagnosis & Pubertal status & Methotrexate & Antiemetic & Outcome \\
\hline Osteosarcoma & Mets & 14 & Midpuberty & $\mathrm{Y}$ & $o+d$ & de \\
\hline Osteosarcoma & No mets & $10.7^{*}$ & Prepuberty & $\mathrm{N}$ & $o+d$ & $a+w$ \\
\hline Osteosarcoma & No mets & $9.7^{*}$ & Prepuberty & $\mathrm{N}$ & $o+d$ & de \\
\hline NHL & Stage 3 & $12.9 *$ & Prepuberty & $\mathrm{Y}$ & $o+p$ & $a+w$ \\
\hline NHL & Stage 3 & $12.7^{*}$ & Prepuberty & $\mathrm{Y}$ & $o+p$ & $a+w$ \\
\hline NHL & Stage 3 & $8.3^{*}$ & Prepuberty & $\mathrm{Y}$ & $o+p$ & $a+w$ \\
\hline Ewings & Mets & 11.4 & Early puberty & $\mathrm{N}$ & $o+d$ & $a+w$ \\
\hline Ewings & No mets & $9.2 *$ & Prepuberty & $\mathrm{N}$ & $o+d$ & $a+w$ \\
\hline NBT & Stage 4 & 2.5 & Prepuberty & $\mathrm{N}$ & $o+d$ & de \\
\hline NBT & Stage 3 & 2.2 & Prepuberty & $\mathrm{N}$ & $o+d$ & $a+w$ \\
\hline NBT & Stage 4 & 2.2 & Prepuberty & $\mathrm{N}$ & $o+d$ & de \\
\hline NBT & Stage 4 & 1.8 & Prepuberty & $\mathrm{N}$ & $o+d$ & de \\
\hline RMS & No mets & 2.3 & Prepuberty & $\mathrm{N}$ & $o+d$ & $a+w$ \\
\hline RMS & No mets & 1.3 & Prepuberty & $\mathrm{N}$ & $o+d$ & $a+w$ \\
\hline Wilms & No mets & 4.5 & Prepuberty & $\mathrm{N}$ & o & $a+w$ \\
\hline Wilms & No mets & 3.5 & Prepuberty & $\mathrm{N}$ & o & $a+w$ \\
\hline GCT & No mets & $8 *$ & Prepuberty & $\mathrm{N}$ & o & $a+w$ \\
\hline Medullo & No mets & 2.6 & Prepuberty & $\mathrm{Y}$ & o & $a+w$ \\
\hline
\end{tabular}

NHL, non-Hodgkin's lymphoma; NBT, neuroblastoma; RMS, rhabdomyosarcoma; GCT, germ cell tumor; medullo, medulloblastom; mets, metastases; o, ondansetron; d, dexamethasone; p, prednisolone; de, deceased; a $+\mathrm{w}$, alive and well.

* Anthropometric studies. 
measurements were made using the random zero method to reduce observer bias (1). Compliance with the use of the knemometer is possible only in children who are older than $5 \mathrm{y}$. Pubertal patients were not measured to avoid the confounding influence of the pubertal growth spurt. One patient attended her local center for follow-up on completion of treatment, so we were unable to obtain further anthropometric indices or blood samples.

All of the patients in the study had central venous access devices to facilitate chemotherapy administration. Blood samples were taken before and after the courses of chemotherapy $(n=25)$ and on completion of treatment $(n=12)$. During treatment, samples were occasionally not obtained if the access device would not bleed back. On completion of treatment, we obtained samples from only those patients who required venipuncture for clinical management. Sampling was performed between $1100 \mathrm{~h}$ and $1500 \mathrm{~h}$ whenever possible to minimize effects of circadian rhythm. Plasma and serum were stored at $-70^{\circ} \mathrm{C}$ until analysis.

\section{Analytical Methods}

We measured procollagen type I C-terminal peptide (P1CP), the cross-linked telopeptide of type 1 collagen (1CTP), and procollagen type III N-terminal propeptide (P3NP) in plasma by RIA (Orion Diagnostica, Espoo, Finland) by methods previously described (11-13). All samples were analyzed in duplicate. As far as possible, all samples were analyzed in a single analytical run to minimize variation. Between-run coefficients of variation were $7.8 \%$ and $5.2 \%$ at 94 and $320 \mu \mathrm{g} / \mathrm{L}$ for P1CP, $6.3 \%$ and $9.2 \%$ at 8.7 and $33.8 \mu \mathrm{g} / \mathrm{L}$ for $1 \mathrm{CTP}$, and $5.6 \%$ and $6.4 \%$ at 4.6 and $10.4 \mu \mathrm{g} / \mathrm{L}$ for P3NP.

Bone alkaline phosphatase (BALP) was measured in plasma by wheat germ lectin affinity electrophoresis (14). Between-run coefficients of variation were $2.2 \%, 3.5 \%$, and $1.9 \%$ at 251 , 349 , and $435 \mathrm{U} / \mathrm{L}$, respectively.

IGF1 was measured in serum with a specific RIA (Mediagnost, Tubingen, Germany) as previously described (15). Between-assay coefficients of variation were $8.5 \%, 6.5 \%$, and $8.0 \%$ at 69,140 , and $118 \mu \mathrm{g} / \mathrm{L}$, respectively.

IGF binding protein 3 (IGFBP3) was measured in serum with a specific RIA (16). Between-assay coefficients of variation were $7.3 \%$ and $6.9 \%$ at 2772 and $3545 \mu \mathrm{g} / \mathrm{L}$, respectively.

IGFBP2 was measured in serum with a specific RIA (17). Recombinant human IGFBP2 (a gift from Sandoz, Basel, Switzerland) was used as a standard and as the tracer. The between-assay coefficient of variation was $10.7 \%$.

\section{Data Analysis}

Anthropometric data. Height SD scores (SDSs) were calculated for comparison within the group (18). Body mass index (BMI) was calculated (weight in $\mathrm{kg} /$ height in $\mathrm{m}^{2}$ ), and SDSs were calculated (19). Both measurements were standardized to allow comparison over time within patient and between patients at diagnosis, on completion of treatment, and on followup. For the knemometry data, we calculated LLL velocity (LLLV) for each time point by subtracting from the LLL at that time point, the length measured at the previous time point, and dividing the difference by the intervening time to achieve a velocity, quoted as $\mathrm{mm} / \mathrm{wk}$. The mean velocity on treatment was compared with the mean value off treatment. Changes in anthropometric data were analyzed by Wilcoxon signed ranks.

Biochemical markers. We expressed all biochemical data as SDSs relative to our own published reference groups to compensate for variation with age and sex and to facilitate comparison with other markers $(7,20-22)$. The distributions of serum concentrations of collagen markers, IGF1, and IGFBPs in healthy children are log normal. We therefore transformed measured concentrations to their logarithms before calculating age- and sex-specific SDSs based on our own published data. BALP did not require log transformation.

We calculated the mean analyte SDS before and after each course of treatment and 2 mo after completion of treatment. Group data were expressed as mean and 95\% confidence intervals for the mean. Variances did not differ significantly among groups, so parametric tests were used throughout. Comparison with the normal reference group (with a mean SDS of 0 ) was by the one-sample $t$ test. Comparisons of measurements between courses and between pre- to posttreatment values were by paired $t$ tests. Relationships between variables were explored using the Pearson correlation coefficient, $r$. Trends during treatment were analyzed by linear regression. All statistical tests were two-tailed, and $p<0.05$ was regarded as significant. All patients received a minimum of six courses of chemotherapy. Statistical analysis of the markers during treatment was therefore restricted to these first six courses.

\section{RESULTS}

\section{Anthropometric Data}

Height SDS $(n=10)$ at diagnosis was $0.49[-1.17$ to 1.66 ; median (range)], at completion of treatment was $0.25(-1.31$ to 1.04$)$, and 3 mo after end of therapy was 0.32 ( -1.32 to 1.37; Fig. 1). There was a significant decrease in height SDS from diagnosis to completion of chemotherapy $(p<0.01)$ followed by a significant increase in height SDS at 3 mo off

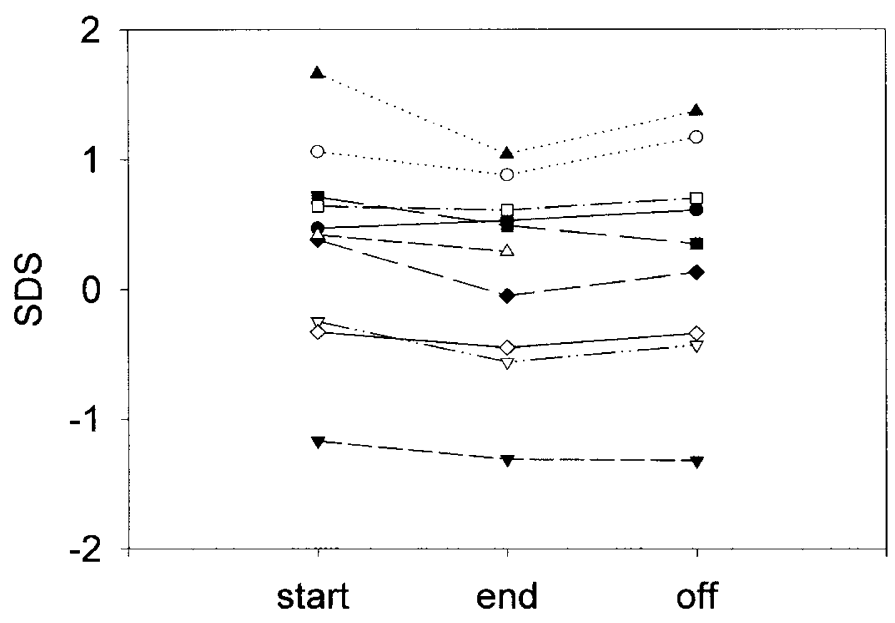

Figure 1. Height SDS at start of treatment, at end of treatment, and 3 mo off treatment in prepubertal children older than $5 \mathrm{y}(n=10)$. (Start $v s$ end, $p<$ 0.01 ; end $v s$ off, $p<0.05$; start $v s$ off, NS.) 
treatment $(p<0.05)$. There was no significant difference between height SDS at diagnosis and 3 mo after completing chemotherapy.

The BMI SDS at diagnosis was 0.1 [ -0.8 to 2.8 ; median (range)], at end of treatment was -0.1 ( -1.4 to 3.2$)$, and 3 mo off treatment was -0.1 ( -0.9 to 3.1 ). There was no significant change in BMI SDS from diagnosis to completion of treatment, and no change in BMI SDS from the end of chemotherapy to reassessment.

LLLV during chemotherapy $(n=10)$ was $0.24 \mathrm{~mm} / \mathrm{wk}$ [ -0.14 to 0.38 ; median (range)] and increased to $0.52 \mathrm{~mm} / \mathrm{wk}$ ( 0.31 to $0.61 ; p<0.01)$ in the 3 mo after completion of treatment $(n=9$; Fig. 2$)$. There was no discernible change in LLLV through treatment.

\section{Bone Markers}

At diagnosis. The mean SDS for each marker at diagnosis is shown in Figure 3. IGF1 and P1CP were significantly lower than the reference group $(p<0.002$ and $p<0.0001$, respectively). BALP was also significantly lower than the reference group $(p<0.0001)$ when the three patients with osteosarcoma were excluded from the analysis. IGFBP2 was significantly elevated $(p<0.001)$. The other markers did not differ significantly from the reference group.

There were positive correlations between IGF1 and IGFBP3 $(r=0.55, p=0.01)$ and between P1CP and 1CTP $(r=0.42$, $p=0.05)$. Negative correlations were observed between IGF1 and IGFBP2 $(r=-0.61, p=0.01)$ and between IGFBP2 and IGFBP3 $(r=-0.71, p=0.01)$.

Comparison of results before and after each course of chemotherapy. $\mathrm{P} 1 \mathrm{CP}, \mathrm{P} 3 \mathrm{NP}$, and $1 \mathrm{CTP}$ all showed highly significant differences comparing the mean SDSs before and after each course of chemotherapy (Fig. $4 A-C$ ). In all three markers, there were significant decreases in SDS after each course of chemotherapy $(p<0.001)$ followed by significant increases before each subsequent course of treatment $(p<$ 0.001). There was no significant change in IGF1, IGFBP3, IGFBP2, or BALP comparing values before and after each course of chemotherapy.

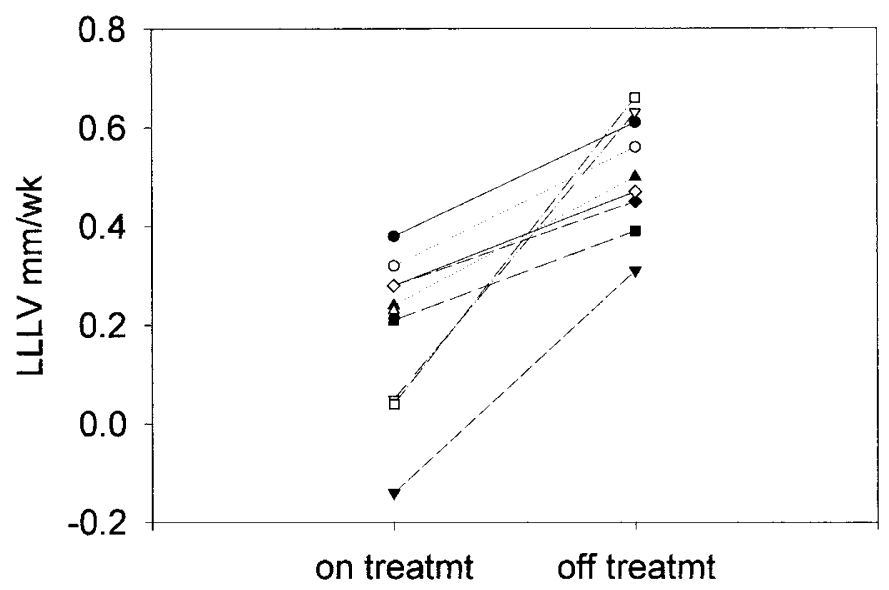

Figure 2. LLLV during treatment compared with 3 mo off treatment $(p<$ $0.01)$.

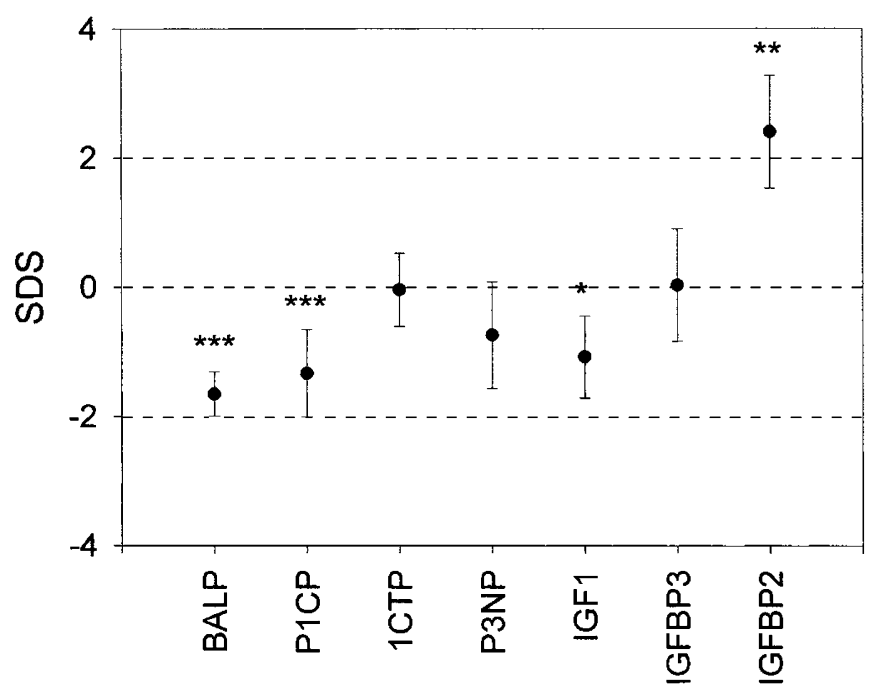

Figure 3. Markers of bone and collagen turnover at diagnosis (BALP: not including osteosarcoma patients; mean, $95 \%$ confidence intervals); ${ }^{*} p<0.01$, ${ }^{* *} p<0.001,{ }^{* * *} p<0.0001$ vs reference population.

Trends throughout treatment. Precourse SDSs for BALP, PICP, ICTP, and P3NP all showed upward trends through treatment $(p<0.001$; Fig. 4A-D). IGF1 $(p<0.05)$ and IGFBP3 $(p<0.01)$ also showed upward trends (Fig. $4 E$ and $f$ ), but IGFBP2 did not change significantly (Fig. $4 G$ ). By contrast, postcourse SDSs for the collagen markers PICP, ICTP, and P3NP showed no significant trend through treatment. Despite its upward trend, BALP remained significantly lower than the reference group throughout treatment $(p<0.0001$; Fig. $4 D$ ). Precourse PICP did not differ significantly from the reference group after the first course of chemotherapy, but postcourse PICP was low compared with the reference group $(p<0.001$; Fig. 4A). Conversely, precourse ICTP was high compared with the reference group $(p<0.01 ;$ Fig. $4 B)$ after the second course of chemotherapy, but postcourse ICTP did not differ significantly from the reference group. Neither pre- nor postcourse P3NP differed significantly from the reference group during the first six cycles of chemotherapy (Fig. 4C).

IGF1 did not differ significantly from the reference group after the second course of chemotherapy (Fig. 4E), but IGFBP3 became significantly elevated compared with the reference group after the first course of chemotherapy $(p<0.01$; Fig. $4 F)$. IGFBP2 remained significantly high compared with the reference group throughout treatment $(p<0.005$; Fig. $4 G)$.

\section{End of Treatment}

The mean SDSs 1 mo after completion of treatment were significantly less than the reference group for BALP $(p<0.05)$ and IGF1 $(p<0.01)$; not significantly different from the mean for P1CP and P3NP; and greater than the mean for 1CTP, IGFBP3, and IGFBP2 $(p<0.001, p<0.001$, and $p<0.01$, respectively). On completion of chemotherapy, there was no significant change in any of the biochemical markers comparing the SDS before the last course of chemotherapy with the values $1 \mathrm{mo}$ off treatment. Comparing the first and second months off treatment values, the only change was an increase in P3NP $(p<0.05)$. 

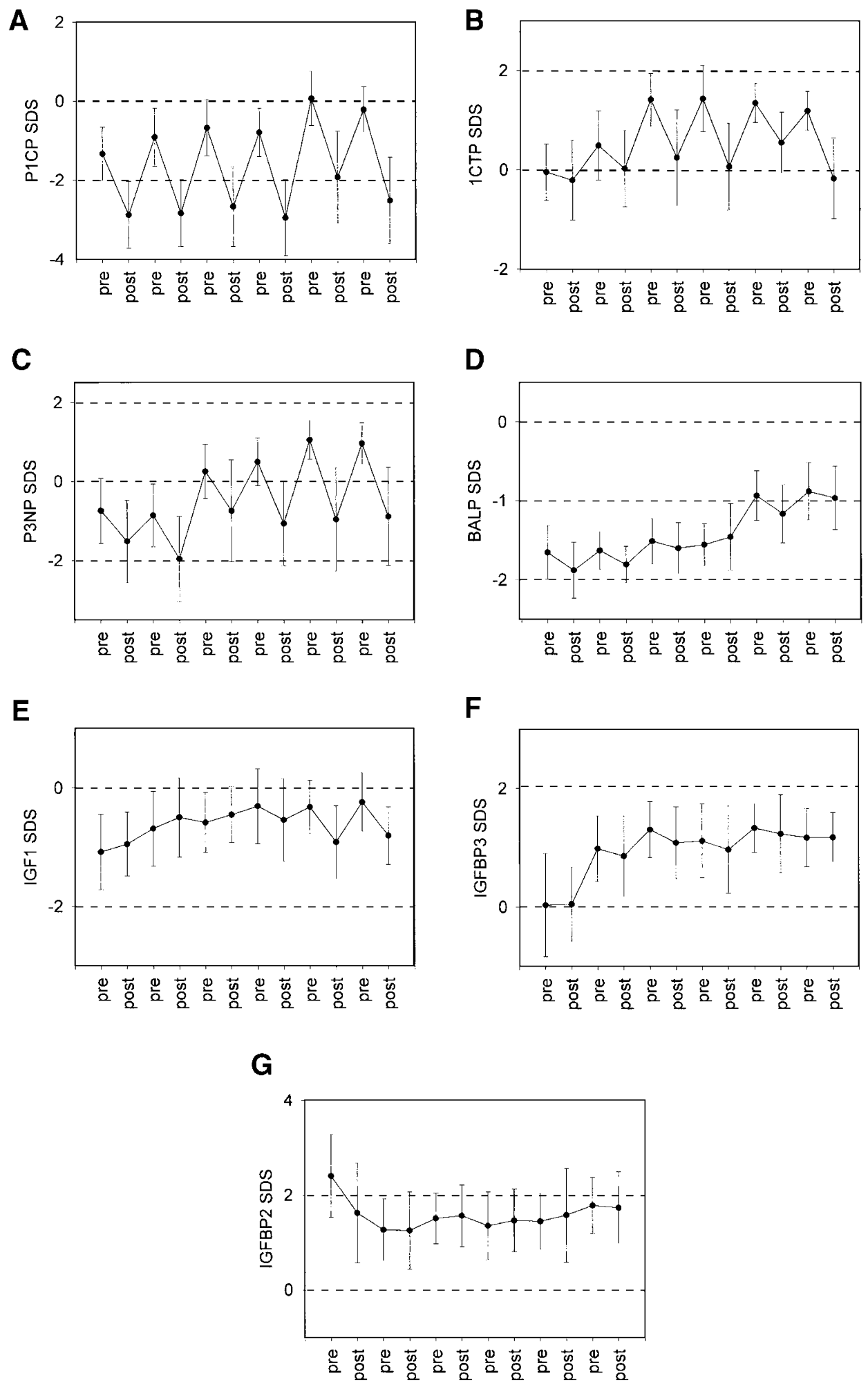

Figure 4. Bone turnover and growth markers before and after each block of chemotherapy. Data expressed as mean and $95 \%$ confidence interval. $(A) \mathrm{P} 1 \mathrm{CP}$. $(B)$ 1CTP. (C) P3NP. (D) BALP (not including osteosarcoma patients). (E) IGF1. (F) IGFBP3. ( $G$ ) IGFBP2.

\section{DISCUSSION}

Growth is mediated via $\mathrm{GH}$, which stimulates the production of IGF1. IGF1, in turn, acts at the growth plate to promote bone formation (23). Circulating IGF1 is largely bound to IGFBP3, which controls its tissue bioavailability. Although the function of IGFBP2 is less well understood, it has been shown to be inversely related to GH secretion (24) and to inhibit IGF1stimulated bone cell proliferation and bone collagen synthesis $(23,25,26)$. There are various biochemical markers that can help elucidate the process of bone turnover (27). P1CP is a 
marker of osteoblast proliferation in its early proliferative stage and reflects type 1 collagen synthesis, largely from bone. BALP is a marker of activity of the differentiated osteoblast. 1CTP reflects the breakdown of type 1 collagen largely derived from bone. P1CP, BALP, and 1CTP all are released into the circulation during the modeling and elongation of bone. P3NP quantitatively reflects type III collagen turnover in soft tissue.

\section{Before treatment}

At diagnosis, bone formation was suppressed with significantly low BALP and P1CP SDSs. The suppression of bone formation at diagnosis may have been mediated through the suppressed IGF1 levels also observed at diagnosis (23). The cause for the IGF1 suppression is not clear. Patients with solid tumors may have been in a GH-resistant state, as has been reported for children with newly diagnosed acute lymphoblastic leukemia (ALL) (4). IGFBP2 SDSs were significantly elevated at diagnosis, as has been previously reported in children with tumors (28). We demonstrated a negative correlation between IGFBP2 and both IGF1 and IGFBP3, and elevated IGFBP2 therefore may have an indirect effect on the growth plate at diagnosis.

\section{During treatment}

During treatment, growth was slow with significant reduction in height SDS from diagnosis to completion of treatment. LLLV during treatment was lower than previously reported in normal healthy children (5) and in children who were undergoing continuing chemotherapy for leukemia (7). Height SDS and LLLV increased on completion of treatment, with a return to pretreatment height SDS after 3 mo off treatment. Slow growth and subsequent catch-up growth during treatment for ALL have been previously reported $(1,2)$. Slow growth during treatment for solid tumors has also been reported, but there was no evidence of catch-up growth on completion of treatment (10). In our study, the low IGF1 found at diagnosis returned to normal soon after starting treatment. We therefore believe that the slow growth during treatment was due to direct effects of chemotherapy on the growth plate not mediated through the $\mathrm{GH}$ axis.

Although BALP showed a steady increase during chemotherapy, it remained significantly suppressed throughout treatment. BALP is produced by the mature osteoblast, and this therefore indicates an adverse effect on osteoblast maturation that was sustained throughout the treatment. Changes in BALP are gradual, and one would not expect to see rapid fluctuations between treatment cycles. By contrast, the markers of collagen formation and breakdown did show a rapid response to interventions that affect collagen metabolism. We demonstrated repeated and consistent effects on these markers throughout the first six courses of chemotherapy.

There was a significant decrease in all three markers of collagen turnover after each course and significant increase before the next course of chemotherapy. P1CP was normal before each course of chemotherapy but was significantly suppressed after each course, indicating an effect on the im- mature osteoblast, with a decrease in formation of type 1 collagen. Conversely 1CTP, a marker of osteoclast activity and type 1 collagen degradation, was significantly elevated before each course of chemotherapy and not significantly different from the reference group after each course of chemotherapy. Thus, there was evidence of a consistent effect on osteoblast and osteoclast activity, with an imbalance between formation and breakdown resulting in a net collagen loss over time. This is further supported by the relatively low levels of BALP throughout treatment.

The precourse values of BALP, PICP, 1CTP, and P3NP showed an upward trend from course 1 to course 6 of chemotherapy, indicating increased bone and collagen turnover with time. However, there was no trend in the postcourse values of the markers of collagen turnover over time, suggesting that each course of treatment had a significant and consistent suppressive effect on bone turnover. Between therapies, there was evidence of increased osteoblast and osteoclast activity. After starting treatment, IGF1 returned to normal. IGFBP3 was positively correlated with IGF1 and remained so throughout treatment. Neither they nor IGFBP2 showed variation with each course of treatment. Therefore, the effects on osteoblast and osteoclast activity during each course of treatment were unlikely to be mediated through the GH axis and were more likely to be due to a direct effect of therapeutic agents.

\section{End of treatment}

The number of patients for whom we obtained serial follow-up samples with sufficient time since completion of chemotherapy was limited, as the majority of children did not require regular blood sampling. BALP and IGF1 were still low 2 mo after completion of treatment, suggesting that osteoblast function was still impaired, possibly mediated through low IGF1 levels. There was an increase in P3NP, which may reflect somatic growth, as was demonstrated by an increase in height on completion of therapy.

The observed effects on the bone markers during treatment were likely to be related to the agents administered. Agents that have been consistently implicated in suppression of bone turnover are methotrexate and steroids $(29-31)$. We suspect that high-dose prednisolone $\left(60 \mathrm{mg} / \mathrm{m}^{2}\right)$ and methotrexate $(3-8$ $\mathrm{mg} / \mathrm{m}^{2}$ ) in the non-Hodkin's lymphoma protocol and high-dose dexamethasone $\left(4 \mathrm{mg} / \mathrm{m}^{2}\right)$ were significant contributors to the changes found in the bone markers. Ahmed et al. (32) reported that the effect of dexamethasone on suppressing linear growth and bone turnover may be 18 and 9 times more, respectively, than prednisolone. We observed similar effects on bone turnover with a prednisolone dose 15 times greater than the dexamethasone dose.

Dexamethasone was not being used as a chemotherapeutic agent but as an antiemetic. There is well-validated research as to its benefit as antiemetic in chemotherapy schedules (33), and the majority of children in our center who are being treated with highly emetogenic protocols receive dexamethasone with good effect. The effect on solid tumor cell lines in vivo is not well reported, but dexamethasone has been shown to suppress osteoblast cell lines in vitro (34), and its use at high dose as an 
antiemetic has a potentially significant effect on bone turnover. High doses of steroids are known to affect bone quality with increased risk of osteoporosis and vertebral collapse. There have been reports of increased bone resorption and reduced bone mineral density during treatment for childhood cancer $(8$, 35 ) and of osteopenia in young adult survivors of childhood cancer (36). We do not have bone mineral density results on our patients because our study was not designed to determine whether there would be long-term effects on bone quality. Prolonged study will be required to address this issue in children who are treated with intensive chemotherapy. The role of dexamethasone as an antiemetic and its effect on tumor cell lines also requires further evaluation.

\section{CONCLUSION}

In summary, we have documented an effect of treatment for solid tumors on short-term growth and bone turnover in children that may partly be attributable to the high-dose dexamethasone that is widely used as an antiemetic during chemotherapy. However, until other therapeutic options are available, its use is likely to continue. Bone mineral density should be assessed in long-term survivors who have received high doses of steroids and methotrexate.

\section{REFERENCES}

1. Ahmed SF, Wallace WHB, Kelnar CKH 1997 An anthropometric study of children during intensive chemotherapy for acute lymphoblastic leukaemia. Horm Res 48:178-183

2. Holm K, Nysom K, Hertz H, Muller J 1994 Normal final height after treatment for acute lymphoblastic leukaemia without irradiation. Acta Paediatr 83:1287-1290

3. Hokken-Koelega ACS, Van Doorn JWD, Hählen K Stijnen T, de Muinck KeizerSchrama SM, Drop SL 1993 Long-term effects of treatment for acute lymphoblastic leukaemia with and without cranial irradiation on growth and puberty: a comparative study. Pediatr Res 33:577-582

4. Crofton PM, Ahmed SF, Wade JC, Stephen R, Elminger MW, Ranke MB, Kelnar CJH, Wallace WHB 1998 Effects of intensive chemotherapy on bone and collagen turnover and the growth hormone axis in children with acute lymphoblastic leukaemia. J Clin Endocrinol Metab 83:3121-3129

5. Ahmed SF, Wallace WHB, Kelnar CJH 1995 Knemometry in childhood: a study to compare the precision of two different techniques. Ann Hum Biol 22:247-252

6. Crofton PM, Ahmed SF, Wade JC, Elmlinger MW, Ranke MB, Kelnar CJH, Wallace WHB 1999 Effects of a third intensification block of chemotherapy on bone and collagen turnover, insulin-like growth factor 1, its binding proteins and short term growth in children with acute lymphoblastic leukaemia. Eur J Cancer 35:960-967

7. Crofton PM, Ahmed SF, Wade JC, Elmlinger MW, Ranke MB, Kelnar CJH, Wallace WHB 2000 Bone turnover and growth during and after continuing chemotherapy in children with acute lymphoblastic leukaemia. Pediatr Res 48:490-496

8. Arikoski P, Komulainen J, Riikonen P, Voutilainen R, Knip M, Kroger H 1999 Alterations in bone turnover and impaired development of bone mineral density in newly diagnosed children with cancer: a 1-year prospective study. J Clin Endocrinol Metab 84:3174-3181

9. Warner JT, Evans WD, Webb DK, Bell W, Gregory JW 1999 Relative osteopenia after treatment for acute lymphoblastic leukaemia. Pediatr Res 45:544-551

10. Roman J, Villaizan CJ, Garcia-Foncillas J, Azcona C, Salvador J, Sierrasesumaga L 1995 Chemotherapy-induced growth hormone deficiency in children with cancer. 25:90-95

11. Mellko J, Niemi S, Risteli L, Risteli J 1990 Radioimmunoassay of the carboxyterminal propeptide of human type I collagen. Clin Chem 36:1328-1332
12. Ristelli J, Elooma I, Niemi S, Novamo A, Risteli L 1993 Radioimmunoassay for the pyrodinoline cross-linked carboxyterminal telopeptide of type I collagen: a new serum marker of bone collagen degradation. Clin Chem 39:635-640

13. Ristelli J, Niemi S, Trivedi P, Maentausta O, Mowat AP, Risteli L 1988 Rapid equilibrium radioimmunoassay for the amino terminal propeptide of human type III procollagen. Clin Chem 34:715-718

14. Peaston RT, Cooper J 1986 Affinity electrophoresis of alkaline phosphatase isoenzymes. Clin Chem 32:235-236

15. Blum WF, Breier BH 1994 Radioimmunoassay for IGFs and IGFBPs. Growth Regul 4(suppl 1):11-19

16. Blum WF, Ranke MB, Kietzmann K, Gauggel E, Zeisel JH, Bierich JR 1990 A specific radioimmunoassay for the growth hormone $(\mathrm{GH})$-dependent somatomedinbinding protein: its use for the diagnosis of growth hormone deficiency. J Clin Endocrinol Metab 70:1292-1297

17. Elmlinger MW, Wimmer K, Biemer E Blum WF, Ranke MB, Dannecker GE 1996 Insulin-like growth factor binding protein 2 (IGFBP-2) is differentially expressed in leukaemic T- and B-cell lines. Growth Regul 6:152-157

18. Freeman JV, Cole TJ, Chinn S, Jones PRM, White EM, Preece MA 1990 Cross sectional stature and weight reference curves for the UK, 1990. Arch Dis Child $73: 17-24$

19. Cole TJ, Freeman JV, Preece MA 1995 Body mass index reference curves for the UK, 1990. Arch Dis Child 73:25-29

20. Crofton PM 1992 Wheat-germ lectin affinity electrophoresis for alkaline phosphatase isoforms in children: age-dependent reference ranges and changes in liver and bone disease. Clin Chem 38:663-670

21. Blum WF 1996 Insulin-like growth factors and their binding proteins. In: Ranke MB (ed) Diagnostics of Endocrine Function in Children and Adolescents. JA Barth, Edition J\&J, Heidelberg, pp 190-218

22. Crofton PM, Wade JC, Taylor MRH, Holland CV 1997 Serum concentrations of the carboxyterminal propeptide of type I procollagen, the aminoterminal propeptide of type III procollagen, the cross-linked carboxyterminal telopeptide of type I collagen and their interrelationships in school children. Clin Chem 43:1577-1581

23. Rosen CJ, Donahue LR, Hunter SJ 2061994 Insulin-like growth factors and bone: the osteoporosis connection. Proc Soc Exp Biol Med 206:83-102

24. Jackson Smith W, Jeong Nam T, Underwood LE, Busby WH, Celnicker A Clemmons DR 1993 Use of insulin-like growth factor binding protein-2 (IGFBP-2), IGFBP-3, and IGF-1 for assessing growth hormone status in short children. J Clin Endocrinol Metab 77:1294-1299

25. Ferry RJ, Cerri RW, Cohen P 1999 Insulin-like growth factor binding proteins: new proteins, new functions. Horm Res 51:53-67

26. Kelley KM, Oh Y, Gargosky SE, Matsumoto T, Hwa V, Ng L, Simpson DM, Rosenfeld RG 1996 Insulin-like growth factor-binding proteins (IGFBPs) and their regulatory dynamics. Int J Biochem Cell Biol 28:619-637

27. Crofton PM, Kelnar CJH 1998 Bone and collagen markers in paediatric practice. Int $\mathrm{J}$ Clin Pract 52:557-565

28. Muller HL, Oh Y, Lehrnbecher T, Blum WF, Rosenfeld RG 1994 Insulin-like growth factor-binding protein-2 concentrations in cerebrospinal fluid and serum of children with malignant solid tumours or acute leukaemia. J Clin Endocrinol Metab 79:428434

29. Nesbit M, Krivit W, Heyn R, Sharp H 1976 Acute and chronic effects of methotrexate on hepatic, pulmonary, and skeletal systems. Cancer 37:1048-1054

30. Tonshoff B, Jux C, Mehls O 1996 Glucocorticoids and growth. In: Kelnar CJH (ed) Bailliere's Clinical Paediatrics-Paediatric Endocrinology. Bailliere Tindall, London, pp 309-311

31. Baron J, Huang Z, Oerter KE, Bacher JD, Cutler GB 1992 Dexamethasone acts locally to inhibit longitudinal bone growth in rabbits. Am J Physiol 263:E489-E492

32. Ahmed SF, Tucker P, Mushtaq T, Wallace AM, Williams DM, Hughes IA 2002 Short-term effects on linear growth and bone turnover in children randomised to receive prednisolone or dexamethasone. Clin Endocrinol 57:185-191

33. The Italian Group for Antiemetic Research 2000 Dexamethasone alone or in combination with ondansetron for the prevention of delayed nausea and vomiting induced by chemotherapy. N Engl J Med 342:1554-1559

34. Kim CH, Cheng SL, Kim GS 1999 Effects of dexamethasone on proliferation, activity, and cytokine secretion of normal human bone marrow stromal cells: possible mechanisms of glucocorticoid-induced bone loss. J Endocrinol 162:371-379

35. Aisenberg J, Hsieh K, Kalaitzoglou G, Whittam E, Heller G, Schneider, Sklar C 1998 Bone mineral density in young adult survivors of childhood cancer. J Pediatr Hematol Oncol 20:241-245

36. Vassilopoulou-Sellin R, Brosnan P, Delpassand A, Zietz H, Klein MJ, Jaffe N 1999 Osteopenia in young adult survivors of childhood cancer. Med Pediatr Oncol 32:272278 